\title{
Effects of lactadherin on plasma D-lactic acid and small intestinal MUC2 and claudin-1 expression levels in rats with rotavirus-induced diarrhea
}

\author{
RUI XU ${ }^{1 *}$, YI-HUI LEI $^{1-3^{*}}$, JUN SHI $^{1 *}$, YI-JUN ZHOU ${ }^{1}$, YING-WEI CHEN $^{2,4}$ and ZHEN-JUAN HE ${ }^{1,2}$ \\ ${ }^{1}$ Department of Neonatology, Xinhua Hospital Affiliated to Shanghai Jiao Tong University School of Medicine; \\ ${ }^{2}$ Department of Perinatal Research Laboratory, Shanghai Institute for Pediatric Research Affiliated to \\ Shanghai Jiao Tong University School of Medicine, Shanghai 200092; ${ }^{3}$ Department of Neonatology, \\ The Second Affiliated Hospital and Yuying Children's Hospital of Wenzhou Medical University, Wenzhou, \\ Zhejiang 325000; ${ }^{4}$ Department of Pediatric Gastroenterology and Nutrition Key Laboratory, Xinhua Hospital \\ Affiliated to Shanghai Jiao Tong University School of Medicine, Shanghai 200092, P.R. China
}

Received November 13, 2014; Accepted November 25, 2015

DOI: $10.3892 /$ etm.2016.3015

\begin{abstract}
The aim of the present study was to investigate the effects of lactadherin on plasma D-lactic acid and small intestinal mucin (MUC) 2 and claudin-1 expression levels in rats with diarrhea induced by rotavirus (RV) infection. A total of 75 seven-day-old healthy Sprague-Dawley rats were randomly divided into the following five groups: Control (C), RV infection (RVI), lactadherin before rotavirus infection (LBRI), lactadherin after rotavirus infection (LARI), and blank (B). On day 4 of artificial feeding, the rats in groups RVI, LBRI and LARI were intragastric administered $1 \times 10^{6}$ PFU RV; whereas the rats in groups $\mathrm{C}$ and $\mathrm{B}$ were intragastrically administered an equal volume of maintenance solution from the RV supernatant and normal saline, respectively. In the LBRI and LARI groups, rats received daily intragastric administration of $0.25 \mathrm{mg}$ lactadherin for three days prior to and following infection with RV, respectively. The course of diarrheal symptoms was observed in each group and samples
\end{abstract}

Correspondence to: Dr Zhen-Juan He, Department of Neonatology, Xinhua Hospital Affiliated to Shanghai Jiao Tong University School of Medicine, 1665 Kongjiang Road, Yangpu, Shanghai 200092, P.R. China

E-mail: hezhenjuan@sjtu.edu.cn

*Contributed equally

Abbreviations: RV, rotavirus; MFG-E8, milk-fat globule-EGF factor 8; OD, optical density; SD, standard deviation; RGD, Arg-Gly-Asp; PBS, phosphate buffered saline; C, control group; RVI, RV infection group; LBRI, lactadherin before rotavirus infection group; LARI, lactadherin after rotavirus infection; $\mathrm{B}$, blank group

Key words: lactadherin, rotavirus infection, neonatal rats, small intestine, barrier were collected on days 1,4 , and 7 post-infection in order to determine the mucosal morphology, plasma D-lactic acid levels and the expression levels of MUC2 and the intracellular junction protein, claudin-1, in the small intestine. On day 4 post-infection, the rats in group RVI demonstrated severely damaged small intestines and typical diarrheal characteristics, as detected by light microscopy; whereas rats in groups LBRI and LARI demonstrated intact small intestinal villi with partial vacuolation of epithelial cells and changes in the position of their nuclei. Electron microscopy demonstrated that the rats in the RVI group had sparse, shortened, disordered intestinal microvilli and widened intercellular junctions; whereas those in groups LBRI and LARI had long intestinal microvilli sparser compared with groups B and C and slightly widened intercellular junctions. Plasma D-lactic acid levels were increased in groups RVI, LBRI and LARI, as compared with groups B and C, and the greatest levels were detected in the RVI group on days 1, 4 and 7 post-infection. In addition to maintaining intestinal permeability, lactadherin enhanced the expression levels of MUC2 and reduced the expression of claudin-1; therefore, further protecting the intestinal epithelial barrier, which may contribute to the prevention and treatment of diarrhea induced by infection with RV.

\section{Introduction}

Rotavirus (RV) infection is the leading cause of diarrheal diseases in infants and children worldwide. In developing countries, RV-induced diarrhea accounts for $20-70 \%$ of diarrheal diseases in hospitalized children $<5$-years of age $(1,2)$. Infection with RV causes an estimated 600,000 deaths per year worldwide, with $>85 \%$ of RV-associated mortality occurring in developing or resource-limited countries (3). This high incidence of RV-induced diarrhea has caused great economic burden.

Lactadherin, also known as human breast antigen 46 or milk-fat globule-EGF factor 8 , is a secreted glycoprotein of the milk-fat globule membrane. In milk, lactadherin func- 
tions as an antiviral protein inhibiting the symptoms of RV infection; however, little is known regarding its physiological function (4-6). Lactadherin binds to ligands in epithelial cells, which may indicate it has a protective function on intestinal cells (5). RV infection is capable of inducing diarrhea via various mechanisms, including the destruction of intestinal epithelial cells, intestinal villus ischemia and enteric nervous system activation (3). The authors of the present study have previously demonstrated that paracellular leakage induced by damage to the intracellular junctions may be associated with the pathogenesis of RV-induced diarrhea (5). Intracellular junctions form part of the intestinal epithelial barrier, and prevent the invasion of microorganisms and their toxins from the intestinal tract (7). Therefore, RV infection may be associated with the intestinal epithelial barrier.

The present study was conducted to investigate the effects of lactadherin on the intestinal epithelial barrier. Various parameters associated with the intestinal epithelial barrier, including plasma D-lactic acid, intestinal mucin (MUC) 2 and intracellular junction claudin-1 protein were investigated in order to investigate the mechanism underlying lactadherin intervention. The results of the present study may provide evidence for the prevention and treatment of patients with $\mathrm{RV}$-associated diarrhea.

\section{Materials and methods}

Reagents and equipment. A group A RV diagnostic kit was purchased from Beijing Cosmos Biological Pharmaceutical Co., Ltd., (Beijing, China), and the plasma D-lactic acid assay kit was purchased from BioVision, Inc. (Milpitas, CA, USA). Rabbit anti-MUC2 polyclonal antibody (ab76774) was purchased from Abcam (Cambridge, UK), and rabbit anti-claudin-1 antibody (NBP1-67515) was purchased from Novus Biologicals, Ltd. (Milan, Italy). SYBR ${ }^{\circledR}$ Premix Ex $\mathrm{Taq}^{\mathrm{TM}}$, ROX reference dye II and PrimeScript ${ }^{\mathrm{TM}}$ reverse transcription (RT) reagent kit were purchased from Takara Biotechnology Co., Ltd. (Dalian, China). Forward and reverse primers and human lactadherin DNA were purchased from Sangon Biotech Co., Ltd., (Shanghai, China).

Laboratory animals and model preparation. Seven-day-old, specific pathogen-free, healthy Sprague-Dawley rats $(n=75)$ were purchased from Shanghai SLAC Laboratory Animal Co., Ltd. (Shanghai, China). Experimental protocols were approved by the ethics committee of Xinhua Hospital Affiliated to Shanghai Jiaotong University School of Medicine (XHEC-F-2014-024; Shanghai, China). Rats were allocated at random into five groups $(n=15$ each), including: Control (C), RV infection (RVI), lactadherin before RV infection (LBRI), lactadherin after RV infection (LARI) and blank (B) groups. Human RV Wa strains were provided by the Institute of Virology of the Chinese Academy of Preventive Medicine (Beijing, China). Rats were intragastrically fed formula milk without lactadherin from seven-days-old (8). On day 4 of artificial feeding, 10-day-old rats in group RVI were intragastrically administered $1 \times 10^{6}$ PFU RV. The LBRI group was intragastrically administered $0.25 \mathrm{mg}$ lactadherin daily for the first three days of artificial feeding and received $1 \times 10^{6} \mathrm{PFU}$ RV, via the same route, on day 4 . The LARI group was intragastrically administered $1 \times 10^{6}$ PFU RV on day 4 of artificial feeding and $0.25 \mathrm{mg}$ lactadherin thereafter for three consecutive days. Group $\mathrm{C}$ received an equal volume of maintenance solution from the RV supernatant (without RV), which contained Dulbecco's modified Eagle's medium supplemented with $1 \mathrm{ug} / \mathrm{ml}$ trypsin (both Gibco; Thermo Fisher Scientific, Inc., Waltham, MA, USA), on day 4 of artificial feeding; whereas group B received an equal volume of normal saline on day 4 of artificial feeding. On days 1, 4 and 7 following RV administration, five rats were randomly selected and anesthetized with $100 \mathrm{mg} / \mathrm{kg}$ ketamine (Fujian Gutian Pharma Co., Ltd., Fujian, China) and $10 \mathrm{mg} / \mathrm{kg}$ diazepam (Jilin Province Tat Animal Pharmaceutical Co., Ltd., Jilin, China) prior to sacrifice via decapitation. Blood samples were harvested and plasma specimens were collected following centrifugation of $0.3-0.5 \mathrm{ml}$ blood at $950 \mathrm{x} \mathrm{g}$ at $4^{\circ} \mathrm{C}$ for $10 \mathrm{~min}$, which was subsequently stored at $-20^{\circ} \mathrm{C}$ prior to D-lactic acid determination. A $5.5-\mathrm{cm}$ section was harvested from the small intestinal tissue along with a $5-\mathrm{cm}$ section via the ileocecal valve, which were immediately washed with phosphate-buffered saline (PBS; Thermo Fisher Scientific, Inc.). Prior to sectioning for electron microscopy, a 2-mm specimen segment was fixed with $2 \%$ glutaraldehyde (Novon Scientific Co., Pleasanton, CA, USA) and a 2-cm specimen segment was frozen in liquid nitrogen and stored at $-80^{\circ} \mathrm{C}$ prior to RT-quantitative polymerase chain reaction ( $\mathrm{qPCR}$ ) analysis. In preparation for the pathological sections, a $2-\mathrm{cm}$ specimen segment was fixed with $10 \%$ neutral formalin buffer (Sinopharm Chemical Reagent Co., Ltd., Shanghai, China).

Lactadherin expression. Lactadherin was provided by Zhangjiang Biotechnology Co. Ltd., (Shanghai, China). The human lactadherin DNA fragment was amplified by PCR from human lactadherin cDNA prior to cloning into the BamHI and EcoRI restriction sites of the pFastBac ${ }^{\mathrm{TM}}$ HTA vector (Invitrogen; Thermo Fisher Scientific Inc.). Once the recombinant bacmid was confirmed, Sf9 insect cells (Zhangjiang Biotechnology Co. Ltd.) were transfected, as previously described (9).

Lactadherin western blot identification and purification. Sf9 cells were detected as polygonal following transfection, as previously described (9), and the supernatant of medium was collected and amplified. Following protein extraction and separation by sodium dodecyl sulfate-polyacrylamide gel electrophoresis, as previously described (9), $50 \mu \mathrm{g}$ protein was incubated with $200 \mu \mathrm{g} / \mathrm{ml}$ rabbit MFG-E8 (H-60) polyclonal immunoglobulin G (1:200; sc-33545; Santa Cruz Biotechnology, Inc., Dallas, TX, USA) at $4^{\circ} \mathrm{C}$ for 12-14 h. Subsequently, the recombinant protein was further purified using S-Sepharose, Q-Sepharose ion-exchange chromatography, and Sephacryl S-100 gel filtration chromatography (GE Healthcare Life Sciences, Shanghai, China), according to the manufacturer's protocol, to $95 \%$ purity, as demonstrated by densitometric scanning (Image Lab Gel Doc $\mathrm{XR}^{+}$system; Bio-Rad Laboratories, Inc., Hercules, CA, USA). The purity and molecular weight of lactadherin were consistent with native lactadherin. 


\section{Diarrhea model establishment}

$R V$ antigen detection in feces. RV antigen rapid detection card (Beijing Cosmos Biological Pharmaceutical Co., Ltd.) was used according to the colloidal gold method (10) with the following criteria: (i) Positive, two red lines; (ii) negative, one control line; and (iii) invalid, no control line, invalid experiment or repetitive detection required.

Feces characteristics. Rat feces were characterized according to four grades as follows: 1, Normal color (brown) and nature; 2, abnormal color (green or yellow-green) and normal nature; 3 , normal color and abnormal nature (thin or watery); and 4 , abnormal color (green or yellow-green) and nature (thin or watery) (11). Diarrhea was diagnosed if the total points were $\geq 2$ (Table I).

Ultraviolet spectrophotometry of enzyme activity. Plasma D-lactic acid levels were determined using a commercial kit according to the manufacturer's protocol. Standard samples of $0,5,10,15,20$ and $25 \mu 1$, were added to a 96-well plate, with the volume adjusted to $50 \mu 1$ using Tris-buffered saline (Thermo Fisher Scientific, Inc.). A total of $50 \mu \mathrm{l}$ plasma specimen was added to the blank wells and $50 \mu$ l enzyme reaction mixture was added to all sample wells, in addition to standard and specimens. Following incubation at room temperature for $30 \mathrm{~min}$, optical density (OD) was measured at $450 \mathrm{~nm}$ using a Synergy ${ }^{\mathrm{TM}} \mathrm{H} 4$ spectrophotometer (BioTek Instruments, Inc., Winooski, VT, USA). Standard curve was obtained in order to calculate plasma D-lactic acid levels as follows: $\mathrm{y}=0.062 \mathrm{x}+0.616$.

Hematoxylin and eosin (HE) staining. In order to examine intestinal mucosal morphology, $5 \mathrm{~cm}$ small intestinal tissue specimens were harvested from the ileocecal valve on day 4 post-infection. Tissue specimens were fixed with $10 \%$ neutral formalin buffer were subjected to gradient ethanol dehydration, xylene transparency, paraffin embedding, sectioning and HE staining (Changdao Biological Technology Co., Ltd., Shanghai, China) prior to light microscopy (Olympus CX21; Corporation, Tokyo, Japan) to observe the morphology of the small intestine mucosa.

Electron microscopy. In order to examine the ultrastructure and intracellular conjunctions of the intestinal epithelial cells, $5 \mathrm{~cm}$ small intestinal tissue specimens were harvested from the ileocecal valve on day 4 post-RV infection. Intestinal tissues were fixed with $2 \%$ glutaraldehyde and pure epoxy resin 618 embedding (China National Chemical Co., Ltd., Beijing, China), sectioning, lead citrate e-dyeing and electron microscopy analysis was performed by the Electron Microscopy Division at Shanghai Jiaotong University School of Medicine.

Immunohistochemical streptavidin-peroxidase analysis. Immunohistochemical analysis of small intestinal MUC2 and intracellular junction claudin-1 protein was performed using intestinal tissue specimens harvested on day 4 post-infection. Tissue specimens were fixed, embedded, sectioned and de-waxed using conventional methods (12) and high-pressure antigen retrieval of specimens was subsequently performed by
Table I. Feces scoring of pups among the five groups at various time points.

\begin{tabular}{llllll}
\hline Day & Control & RVI & LBRI & LARI & Blank \\
\hline D1 & $1.0 \pm 0.0^{\mathrm{a}}$ & $2.0 \pm 0.5^{\mathrm{b}}$ & $1.0 \pm 0.5^{\mathrm{a}, \mathrm{b}}$ & $2.0 \pm 0.5^{\mathrm{b}}$ & $1.0 \pm 0.0^{\mathrm{a}}$ \\
D2 & $1.0 \pm 0.0^{\mathrm{a}}$ & $3.0 \pm 1.0^{\mathrm{b}}$ & $2.0 \pm 0.5^{\mathrm{a}, \mathrm{b}}$ & $2.0 \pm 1.0^{\mathrm{a}, \mathrm{b}}$ & $1.0 \pm 0.0^{\mathrm{a}}$ \\
D3 & $1.0 \pm 0.0^{\mathrm{a}}$ & $3.0 \pm .0 .5^{\mathrm{b}}$ & $2.0 \pm 1.0^{\mathrm{a}, \mathrm{b}}$ & $2.0 \pm 0.5^{\mathrm{a}, \mathrm{b}}$ & $1.0 \pm 0.0^{\mathrm{a}}$ \\
D4 & $1.0 \pm 0.0^{\mathrm{a}}$ & $4.0 \pm 1.0^{\mathrm{b}}$ & $3.0 \pm 1.0^{\mathrm{a}, \mathrm{b}}$ & $3.0 \pm 0.0^{\mathrm{a}, \mathrm{b}}$ & $1.0 \pm 0.0^{\mathrm{a}}$ \\
D5 & $1.0 \pm 0.0^{\mathrm{a}}$ & $3.0 \pm 0.5^{\mathrm{b}}$ & $1.0 \pm 1.0^{\mathrm{a}}$ & $2.0 \pm 1.0^{\mathrm{a}}$ & $1.0 \pm 0.0^{\mathrm{a}}$ \\
D6 & $1.0 \pm 0.0^{\mathrm{a}}$ & $3.0 \pm 0.5^{\mathrm{b}}$ & $1.0 \pm 0.5^{\mathrm{a}}$ & $1.0 \pm 1.0^{\mathrm{a}}$ & $1.0 \pm 0.0^{\mathrm{a}}$ \\
D7 & $1.0 \pm 0.0$ & $1.0 \pm 1.0^{\mathrm{b}}$ & $1.0 \pm 0.0$ & $1.0 \pm 0.0$ & $1.0 \pm 0.0$ \\
\hline
\end{tabular}

Data are presented as the median \pm quartile interval, where the quartile interval is the degree of dispersion. ${ }^{\mathrm{a}} \mathrm{P}<0.05$ vs. the RVI group; ${ }^{\mathrm{b}} \mathrm{P}<0.05$ vs. the control group. RVI, rotavirus infection; LBRI, lactadherin before RVI; LARI, lactadherin after RVI.

incubating the specimens in $3 \% \mathrm{H}_{2} \mathrm{O}_{2}$ at room temperature for 10 min. Subsequently, the specimens were soaked twice in PBS for $5 \mathrm{~min}$, and incubated with normal goat serum (Shanghai Haoran Biological Technology Co., Ltd., Shanghai, China) at room temperature for $20 \mathrm{~min}$. The serum was discarded and the specimens were subsequently incubated with rabbit primary polyclonal anti-MUC2 (1:100) and anti-claudin-1 (1:100) antibodies $4^{\circ} \mathrm{C}$ overnight. Following this, the specimens were rinsed three times with PBS for $5 \mathrm{~min}$ and incubated with horseradish peroxidase-conjugated goat anti-rabbit secondary antibody (1:100; 111-035-003; Jackson ImmunoResearch Laboratories, Inc., West Grove, PA, USA) working solution at room temperature for $60 \mathrm{~min}$. Specimens were subsequently rinsed three times with PBS for $5 \mathrm{~min}$ and developed using 3,3'-diaminobenzidine reagent (Wuhan Boster Biological Technology, Ltd., Wuhan, China). Hematoxylin counterstaining was performed and the specimens were mounted using the conventional method (12). Five fields of view were randomly selected and images of the intestinal mucosal layer were captured using a light microscope (magnification, x40). Image Pro Plus 6.0 (Media Cybernetics, Inc., Rockville, MD, USA) was used to compare the average OD of the brown yellow particles amongst the various rat groups, which was calculated according to the area of brown yellow particles and their OD values, as follows: $\mathrm{A}=\mathrm{OD} /$ area.

$R T-q P C R$. The mRNA expression levels of intestinal MUC2 mucin and intracellular junction claudin-1 protein expression levels were analyzed using RT-qPCR, using the following primers: MUC2, forward 5'-AGACCGTAGTGCTGTTGA CTGA-3', and reverse 5'-GGTAGGAGGAGGGTTTGA AGAT-3'; claudin-1, forward 5'-AAAAGATGTGGATGG CTGTCA-3', and reverse 5'-GTGGTGTTGGGTAAGAGG TTGT-3'; and $\beta$-actin, forward 5'-CCCATCTATGAGGGT TACGC-3' and reverse 5'-TTTAATGTCACGCACGATTTC-3'. Total RNA was extracted using TRIzol ${ }^{\circledR}$ (Invitrogen; Thermo Fisher Scientific, Inc.) and $0.2 \mathrm{ml}$ chloroform (Sinopharm Chemical Reagent Co., Ltd.) per sample. Following incubation at $37^{\circ} \mathrm{C}$ for $15 \mathrm{~min}$, the $100 \mu \mathrm{g}$ RNA was reverse-transcribed to cDNA at $85^{\circ} \mathrm{C}$ for $5 \mathrm{sec}$ using a Veriti 96-well thermal cycler 


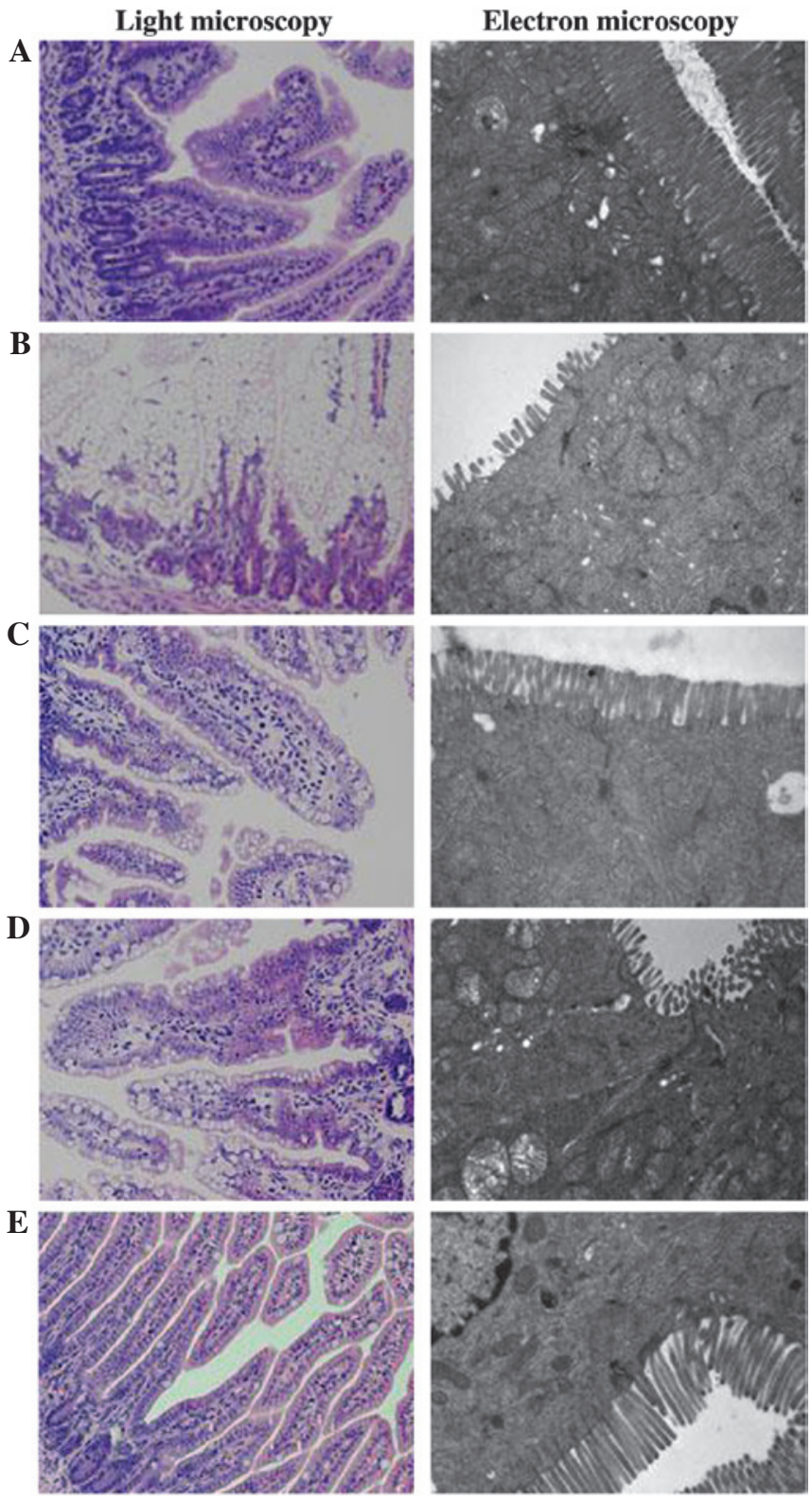

Figure 1. Microphotographs demonstrating alterations in the mucosal morphology of the small intestines of all groups on day 4 following infection with rotavirus (RV). (A) Control; (B) RV infection (RVI); (C) lactadherin before RV infection (LBRI); (D) lactadherin after RV infection (LARI); and (E) blank (B) groups, captured using light (magnification, $\mathrm{x} 200$ ) and electron microscopy (magnification, x17,500).

(Applied Biosystems; Thermo Fisher Scientific, Inc.). To a final volume of $20 \mu 1$, the PCR reaction mixture contained $10 \mu 11 \mathrm{X}$ SYBR Premis Ex Taq $\mathrm{II}^{\mathrm{TM}}, 0.8 \mu 1$ forward primer $(0.4 \mu \mathrm{M}), 0.8 \mu 1$ reverse primer $(0.4 \mu \mathrm{M}), 0.4 \mu 1 \mathrm{1X}$ ROX reference dye II, $2 \mu 1$ DNA template and $6 \mu 1$ RNase-free $\mathrm{dH}_{2} \mathrm{O}$. Amplification conditions were as follows: 1 cycle of $95^{\circ} \mathrm{C}$ for $30 \mathrm{sec}$; followed by 40 cycles of $95^{\circ} \mathrm{C}$ for $5 \mathrm{sec}$ and $60^{\circ} \mathrm{C}$ for $34 \mathrm{sec}$; and one cycle of $95^{\circ} \mathrm{C}$ for $15 \mathrm{sec}, 60^{\circ} \mathrm{C}$ for $1 \mathrm{~min}$, and $95^{\circ} \mathrm{C}$ for $15 \mathrm{sec}$. Results were automatically computed and analyzed using ABI 7500 software (Applied Biosystems; Thermo Fisher Scientific, Inc.). cDNA expression levels were transformed to mRNA expression level, and the sample values were normalized against the $\beta$-actin reference values.

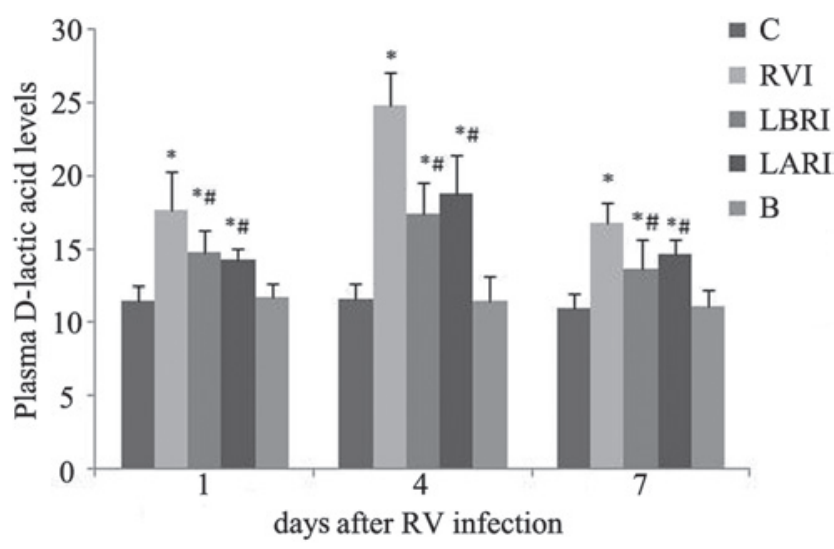

Figure 2. Plasma D-lactic acid levels on days 1, 4 and 7 following RV infection. ${ }^{*} \mathrm{P}<0.05$ vs. the $\mathrm{C}$ group at the same time point; ${ }^{~} \mathrm{P}<0.05$ vs. the RVI group. RV, rotavirus; $\mathrm{C}$, control; RVI, RV infection; LBRI, lactadherin before $\mathrm{RV}$ infection; LARI, lactadherin after RV infection; $\mathrm{B}$, blank.

Statistical methods. Data were analyzed using the SAS statistical software package (version 8.02; SAS Institute Inc., Cary, $\mathrm{NC}$, USA), and presented as the mean \pm standard deviation. Multi-group and pairwise comparisons were performed using single factor analysis of variance and Student-Newman-Keuls tests, respectively. $\mathrm{P}<0.05$ was considered to indicate a statistically significant difference.

\section{Results}

Influence of lactadherin on the course and degree of diarrhea. The feces of rats in groups B and C demonstrated negative RV antigen detection results, which was consistent with their lack of diarrheal symptoms. Positive results were detected in groups RVI, LBRI and LARI, which was consistent with their clinical symptoms of diarrhea $24 \mathrm{~h}$ after intragastric administration of RV, including increased water content or abnormal fecal color. The severity of diarrhea peaked 4 days after intragastric administration of RV.

Among the five rat groups, the most severe clinical symptoms of diarrhea were detected in the RVI group, including substantially increased fecal water content, high defecation frequency and long disease duration, with normal feces predominantly observed by day 7. By comparison, groups LBRI and LARI demonstrated less severe clinical symptoms of diarrhea, including slightly increased fecal water content and short disease duration, with normal feces predominantly observed by day 6 .

Influence of lactadherin on mucosal morphology in rat small intestine. At 4 days post-RV infection, rats in groups $\mathrm{C}$ and $\mathrm{B}$ exhibited intact intestinal villi without swelling, degeneration, necrosis or inflammatory cell infiltration, as detected by light microscopy (Fig. 1A and E). By contrast, swelling and congested intestinal villi with fractures, substantial vacuolation of epithelial cells, changes in the position of the nucleus, non-obvious inflammatory cell infiltration and minimal lymphocytic infiltration was detected in the RVI group (Fig. 1B). In the LBRI and LARI groups, light microscopy demonstrated relatively intact intestinal villi with partial vacuolation of epithelial cells and partial alterations in the position of the nucleus (Fig. 1C and D). 

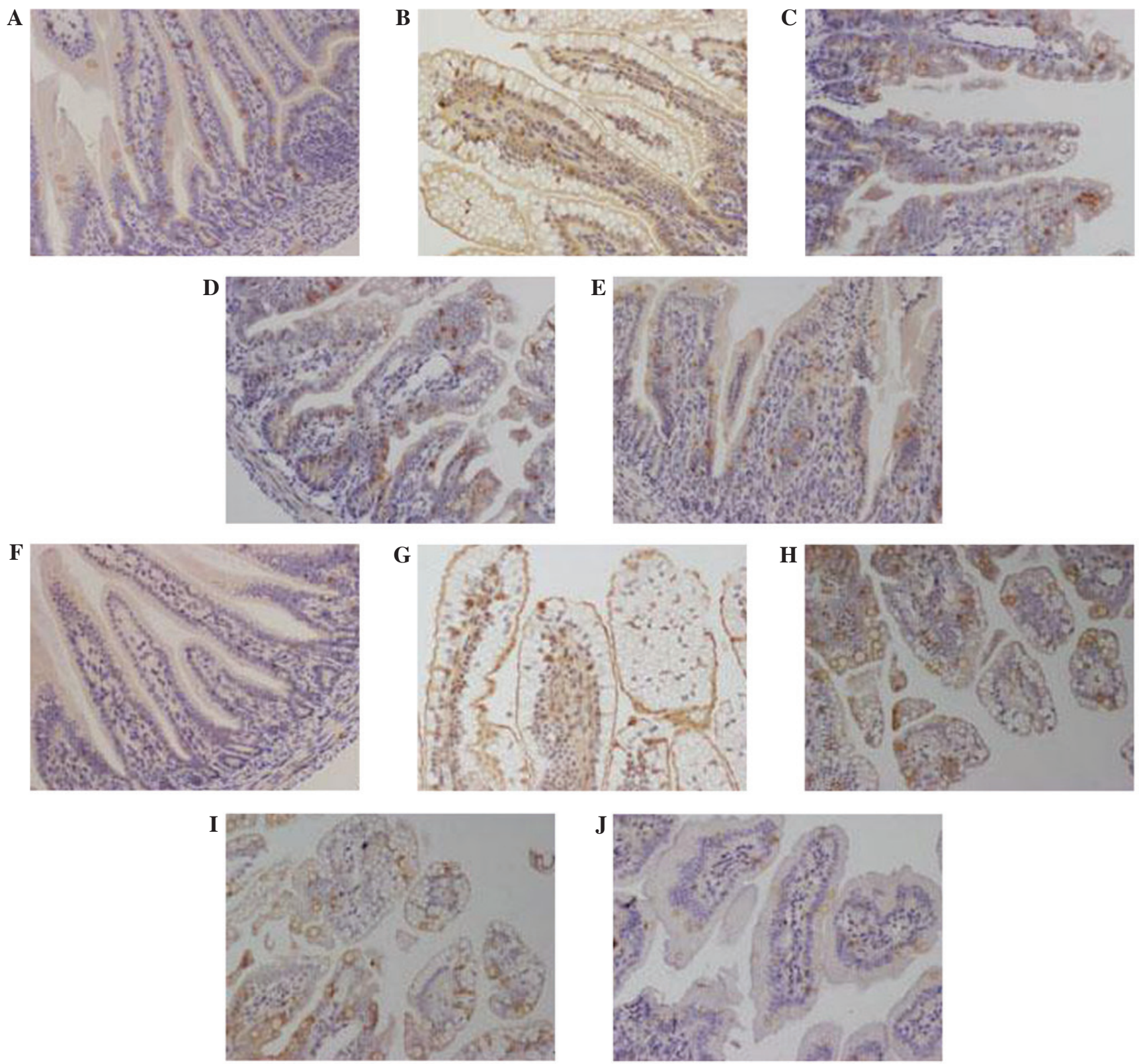

Figure 3. Immunohistochemistry of mucin 2 (MUC2) and claudin-1 on day 4 following rotavirus (RV) infection. Using a light microscope, (A-E) MUC2 expression was predominantly detected in the perinuclear area of the cytoplasm and the nucleus; whereas (F-J) claudin-1 expression was predominantly detected in the cell membrane. (A and F) Control (C); (B and G) RV infection (RVI), (C and H) lactadherin before RV infection (LBRI), (D and I) lactadherin after RV infection (LARI); and (E and J) blank (B) groups (magnification, 200x).

A

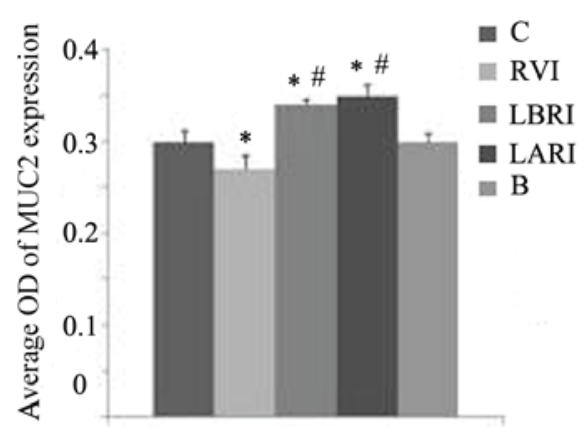

B

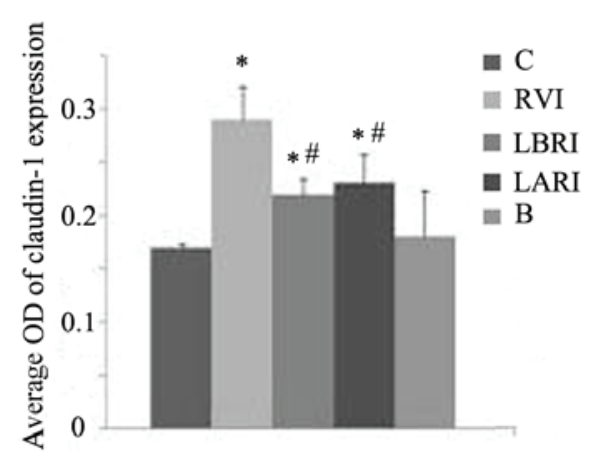

Figure 4. Average OD of the rat groups with positive (A) MUC2 and (B) claudin-1 expression levels. "P<0.05 vs. the C group at the same time point; ${ }^{\text {"P }}<0.05$ vs. the RVI group. OD, optical density; MUC2, mucin 2; C, control; RVI, rotavirus infection; LBRI, lactadherin before RVI; LBRI, lactadherin after RVI; B, blank. 

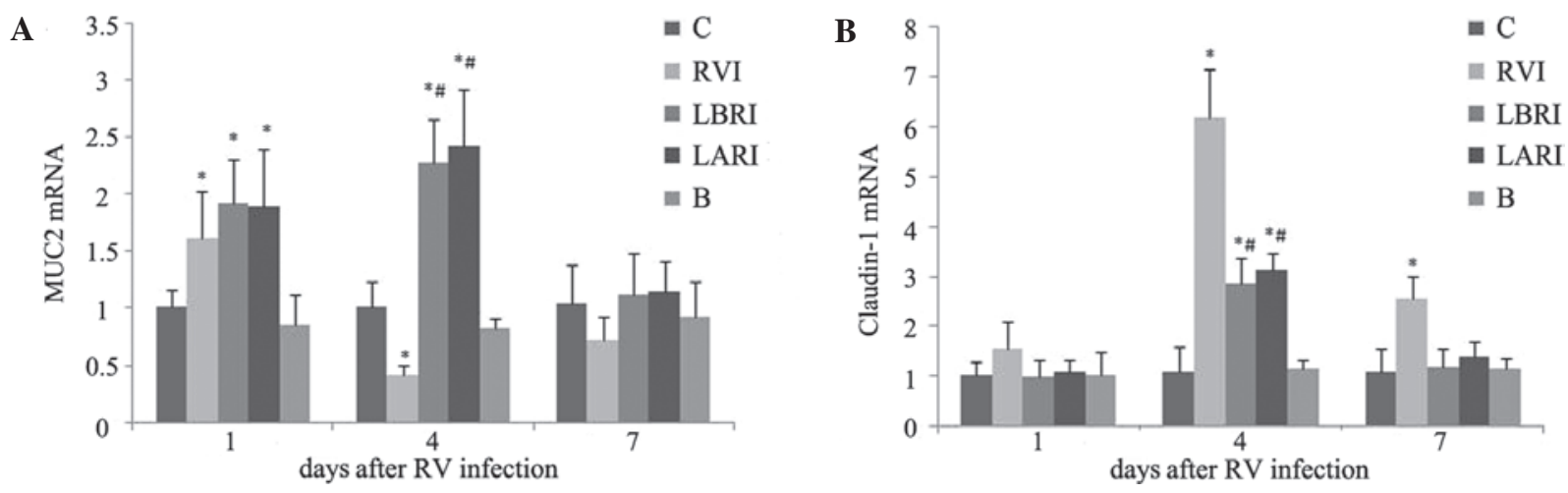

Figure 5. Small intestinal (A) mucin 2 (MUC2) and (B) claudin-1 mRNA expression levels on days 1, 4 and 7 post-infection. ${ }^{*} \mathrm{P}<0.05$ vs. group C at the same time point; and ${ }^{*} \mathrm{P}<0.05$ vs. the RVI group. MUC, mucin; C, control; RVI rotavirus infection; LBRI, lactadherin before RVI; LARI, lactadherin after RVI; B, blank.

As detected by electron microscopy, groups $\mathrm{C}$ and $\mathrm{B}$ demonstrated compact, neat and long intestinal microvilli with closed intracellular junctions at 4 days post-RV infection (Fig. 1A and E); as compared with the sparse, shortened and disordered intestinal microvilli with broadened intracellular junctions and swollen mitochondria detected in the RVI group (Fig. 1B). As compared with groups B and C, groups LBRI and LARI demonstrated longer intestinal microvilli with slightly broadened intercellular junctions and swollen mitochondria (Fig. 1C and D).

Influence of lactadherin on rat plasma D-lactic acid levels. On days 1, 4 and 7 following RV infection, plasma D-lactic acid levels were significantly elevated in groups RVI, LBRI and LARI, as compared with group C $(\mathrm{P}<0.05)$. Notably, plasma D-lactic acid levels were highest in the RVI group, particularly on day 4 post-infection (Fig. 2).

Effects of lactadherin on MUC2 and claudin-1 expression levels in rat small intestine. In groups $\mathrm{C}$ and $\mathrm{B}$ at 4 days post-RV infection, MUC2 expression was evenly distributed (Fig. 3A and E) with no substantial variations in the average OD values (Fig. 4A). In the RVI group, MUC2 expression was predominantly detected in the perinuclear space and cytoplasm with low expression levels detected in the nucleus (Fig. 3B); whereas MUC2 expression was concentrated to specific areas in groups LBRI and LARI (Fig. 3C and D). Furthermore, significantly increased average OD values were demonstrated in the LBRI and LARI groups, as compared with groups $\mathrm{B}$ and $\mathrm{C}(\mathrm{P}<0.05)$. The lowest average $\mathrm{OD}$ was detected in the RVI group, as compared with the other groups (Fig. 4A).

In groups $\mathrm{C}$ and $\mathrm{B} 4$ days post-RV infection, claudin-1 expression was predominantly detected in the cell membrane (Fig. 3F and J), with no differences in the average OD values (Fig. 4B). In groups RVI, LBRI and LARI, claudin-1 expression was predominantly detected in the cell membrane, with low expression levels detected in the nucleus and cytoplasm (Fig. 3G-I). Claudin-1 expression was greatest in the RVI group. The associated average OD values were increased in groups RVI, LBRI and LARI, as compared with groups B and $\mathrm{C}$, with the greatest values detected in the RVI group (Fig. 4B).
Results of MUC2 and claudin-1 mRNA assays. On day 1 post-RV infection, MUC2 mRNA expression levels were significantly increased in groups RVI, LBRI and LARI, as compared with groups $\mathrm{B}$ and $\mathrm{C}(\mathrm{P}<0.05)$. On day 4 post-infection, MUC2 mRNA expression levels were elevated in groups LBRI and LARI and group RVI had significantly decreased, as compared with groups B and C. By day 7 post-infection, no substantial differences in MUC2 mRNA expression levels were detected amongst the various groups (Fig. 5A).

No substantial differences in claudin-1 mRNA expression levels were detected amongst the various groups on day 1 post-infection. However, on day 4 post-infection, claudin-1 mRNA expression levels were significantly increased in groups RVI, LBRI and LARI, as compared with groups B and C $(\mathrm{P}<0.05)$. Claudin-1 mRNA expression levels were highest in the RVI group. On day 7 post-infection, claudin-1 mRNA expression levels were significantly elevated in the RVI group, as compared with the other groups $(\mathrm{P}<0.05)$ (Fig. 5B).

\section{Discussion}

The RV genus belongs to the Reoviridae family, the members of which have genomes containing 11 segments of double-stranded (ds)RNA and primarily infect infants $<2$ years old (13). Lactadherin is crucially involved in reducing the incidence of RV-associated diarrhea and relevant diarrheal symptoms $(4,14,15)$. Previous studies have demonstrated that breast milk contains lactadherin with a specific sequence, i.e., $\mathrm{N}^{\prime}$-Arg-Gly-Asp (RGD) crosslinked with integrin $\alpha_{\mathrm{v}} \beta_{3}$ and $\alpha_{\mathrm{V}} \beta_{5}$, thus competitively inhibiting the RV combination with host cells $(12,14-17)$. The results of the present study demonstrated that, following infection with RV, rats in the groups treated with lactadherin, LBRI and LARI, exhibited less severe diarrheal symptoms, as compared with the RVI group. Furthermore, light and electron microscopy analysis demonstrated that rats in the LBRI and LARI groups suffered less damage to intestinal tissue, as compared with the RVI group, suggesting that lactadherin may have a role in the prevention and treatment of RV-associated diarrhea.

It has previously been demonstrated that intestinal mucosal permeability increases prior to the substantial changes in intestinal mucosal morphology $(18,7)$. Therefore, intestinal 
mucosal permeability reflects early damage to the intestinal mucosa barrier, and D-lactic acid levels may be used to quantitatively assess intestinal mucosal barrier function $(18,7)$. The present study demonstrated that D-lactic acid levels were elevated in rats in groups RVI, LBRI and LARI, as compared with those in groups B and C on days 1, 4 and 7 following $\mathrm{RV}$ infection. RV infection may have caused damage to the intestinal mucosal barrier and increased intestinal permeability, allowing the transport of bacterially produced D-lactic acid from the intestine to the plasma via the damaged mucosa, leading to increased plasma D-lactic levels. D-lactic acid levels peaked on day 4 post-infection, suggesting that the intestinal lesions became most severe at this point, which is consistent with the peak of clinical diarrheal symptoms. Furthermore, plasma D-lactic acid levels decreased in groups LBRI and LARI, as compared with the RVI group. Analyses of the mucosal morphology of the rat's small intestines using light and electron microscopy demonstrated that the damage to the small intestinal tissues was less severe in the LBRI and LARI groups, as compared with the RVI group on day 4 post-infection. These results suggested that lactadherin may have counteracted the increased intestinal permeability induced by RV infection.

The intestinal epithelial barrier is the first-line of mucosal immune defense, and includes the mucus layer, intestinal epithelial cell layer and the tight junctions of intestinal epithelial cells, which are associated with intestinal permeability (19). Previous studies have demonstrated that the intestinal mucus layer, in particular mucin, is able to inhibit the adhesion of viruses to intestinal epithelial cells $(11,20,21)$. To date, 21 mucins have been discovered, of which MUC2, which is most abundant in the small intestine, is one of the most important (22). The mucus gel later is an integral structural component of the intestine, which provides a medium for protection, lubrication and transport between the luminal contents and the epithelial lining (23). As such, as a structural component of the protective mucus layer, MUC2 ensures intestinal mucus has a high density and viscoelasticity and the synthesis rate of MUC2 is a potential parameter for intestinal barrier function (24). Boshuizen et al (22) have previously demonstrated that MUC2 mRNA expression levels were reduced in the jejunums of RV-infected rats, which increased in the ilea of the rats on day 1 post-infection but remained at similar levels at other time points, as compared with the control. The results of the present study demonstrated that on day 1 post-infection, small intestinal MUC2 mRNA expression levels were increased in the rats in groups RVI, LBRI and LARI, as compared with those in groups B and C. The dsRNA and VP4 outer capsid protein of RV may have respectively activated corresponding signal transduction pathways for the direct or indirect upregulation of MUC2 expression (22); therefore, further improving the capability of the immune system to defend against infection. On day 4 post-infection, the mRNA expression levels of MUC2 in the RVI group were reduced compared with those detected in groups $\mathrm{B}$ and $\mathrm{C}$, which may have been due to a reduction in MUC2 secretion caused by a decline of the body's MUC2 reserve and the apoptosis of goblet cells, which are capable of secreting MUC2. Regarding the increased MUC2 mRNA expression levels detected in groups LBRI and LARI, as compared with groups $\mathrm{B}$ and $\mathrm{C}$, it is hypothesized that lactadherin promoted intestinal epithelial cell growth and goblet cell maturation, thus reducing the apoptosis of intestinal epithelial cells. MUC2 is a crucial component of the intestinal epithelial barrier which protects against microbial infection; therefore, this increase in MUC2 expression levels may enhance the defensive function of the intestinal epithelial barrier. This may explain why rats in the LBRI and LARI groups exhibited reduced intestinal permeability following lactadherin administration, which counteracted the increased intestinal permeability induced by infection with RV. Following the removal of inflammatory lesions and the recovery of epithelial cells, MUC2 expression levels should return to normal; however, the underlying mechanisms require further study.

The integrity of the intestinal epithelial barrier is maintained by intestinal epithelial cells and intracellular junctions, which ensure that the areas between two cells are impermeable (25-27). Previous studies have demonstrated that paracellular leakage caused by damage to the intracellular junctions may be an important mechanism underlying RV-associated diarrhea $(20,28)$. Tight junctions have a key role in the association of cells $(25-27,29)$ and in recent years, claudin-1 has been proposed as an important component of the tight junctions in the intestinal barrier $(30,31)$. However, claudin-1 expression levels increase, rather than decrease, with increasing inflammatory intestinal permeability $(25,26)$. In the present study, no substantial variations in claudin-1 mRNA expression levels were detected amongst the rat groups receiving various treatments on day 1 post-RV infection. However, claudin-1 mRNA expression levels were elevated in the RVI, LBRI and LARI groups, as compared with groups B and C; furthermore, the RVI group demonstrated increased levels on day 4 post-infection, as compared with the LBRI and LARI groups. On day 7 post-infection, claudin-1 mRNA expression levels increased in the rats in group RVI, as compared with the other groups. Furthermore, claudin-1 mRNA expression was predominantly detected in the cell membrane; however, low expression levels were detected in the cytoplasm and nuclei of rats in the RVI, LBRI and LARI groups. These results were consistent with a previous study investigating inflammatory bowel disease, conducted by Poritz et al (32). Furthermore, the present study demonstrated that claudin-1 mRNA expression levels were increased in the RVI, LBRI and LARI groups, as compared with groups $\mathrm{B}$ and $\mathrm{C}$ on day 4 post-infection; whereas these levels were increased in the RVI group, as compared with groups B and C on day 7 post-infection. Similarly, plasma D-lactic acid levels were elevated in the RVI, LBRI and LARI groups, as compared with groups B and $\mathrm{C}$ on days 4 and 7 . Claudin- 1 may function as a channel protein in the regulation of tight junctions and its upregulation is likely to have increased the permeability between epithelial cells, resulting in increased intestinal permeability in the rats in groups RVI, LBRI and LARI. Furthermore, the claudin-1 mRNA expression and plasma D-lactic acid levels were reduced in the LBRI and LARI groups, as compared with the RVI group on days 4 and 7 following infection with RV. These results suggested that lactadherin may reduce the upregulated expression of claudin-1 mRNA associated with RV infection in order to maintain the intestinal epithelial barrier and improve intestinal permeability. 
In conclusion, the present study demonstrated that lactadherin increased the expression levels of small intestinal MUC2 and reduced the expression levels of claudin-1 intracellular junction protein, thus protecting the intestinal epithelial barrier and counteracting the increased intestinal permeability caused by infection with RV. These results suggested that lactadherin may be used in the prevention and treatment of RV-associated diarrhea.

\section{Acknowledgements}

The authors of the present study would like to thank Dr Tongxin Chen at the Shanghai Children's Medical Center Affiliated to Shanghai Jiao Tong University School of Medicine (Shanghai, China) for technical support; Professor Luying Peng at Tongji University School of Medicine and Life Science Experimental Teaching Center of Pathogenic Biology Laboratory (Shanghai, China) for providing P2 laboratory; Dr Zhaojun Duan at the China Disease Prevention and Control Center for Disease Prevention Control (Beijing, China) and Professor Haimei Tian at Peking Union Medical College Tumor Hospital (Beijing, China) for providing experimental materials; and Dr Haiqin Shen at Shanghai Jiao Tong University School of Medicine for data collection.

\section{References}

1. Walker CL, Rudan I, Liu L, Nair H, Theodoratou E, Bhutta ZA, O'Brien KL, Campbell $\mathrm{H}$ and Black RE: Global burden of childhood pneumonia and diarrhoea. Lancet 381: 1405-1416, 2013.

2. Lanata CF, Fischer-Walker CL, Olascoaga AC, Torres CX, Aryee MJ and Black RE; Child Health Epidemiology Reference Group of the World Health Organization and UNICEF: Global causes of diarrheal disease mortality in children $<5$ years of age: A systematic review. PLoS One 8: e72788, 2013.

3. Chen SC, Tan LB, Huang LM and Chen KT: Rotavirus infection and the current status of rotavirus vaccines. J Formos Med Assoc 111: 183-193, 2012.

4. Kvistgaard AS, Pallesen LT, Arias CF, López S, Petersen TE, Heegaard CW and Rasmussen JT: Inhibitory effects of human and bovine milk constituents on rotavirus infections. J Dairy Sci 87: 4088-4096, 2004.

5. Bu HF, Zuo XL, Wang X, Ensslin MA, Koti V, Hsueh W, Raymond AS, Shur BD and Tan XD: Milk fat globule-EGF factor 8/lactadherin plays a curcial role in maintenance and repair of murine intestinal epithelium. J Clin invest 117: 3673-3683, 2007.

6. Kusunoki R, Ishihara S, Aziz M, Oka A, Tada Y and Kinoshita Y: Role of milk fat globule-epidermal growth factor 8 in intestinal inflammation. Digestion 85: 103-107, 2012.

7. Ciarlet $\mathrm{M}$ and Estes $\mathrm{MK}$ : Interactions between rotavirus and gastrointestinal cells. Curr Opin Microbiol 4: 435-441, 2001.

8. Kanno T, Koyanagi N, Katoku Y, Yonekubo A, Yajima T, Kuwata T, Kitagawa H and Harada E: Simplified preparation of a refined milk formula comparable to rat's milk: Influence of the formula on development of the gut and brain in artificially reared rat pups. J Pediatr Gastroenterol Nutr 24: 242-252, 1997.

9. Lu H, Zhu J, Zang Y, Ze Y, Qin J: Cloning, high level expression of human paraoxonase-3 in Sf9 cells and pharmacological characterization of its product. Biochem Pharmacol 70:1019-1025, 2005.

10. Kaito M1, Ishida S, Tanaka H, Horiike S, Fujita N, Adachi Y, Kohara M, Konishi M and Watanabe S: Morphology of hepatitis C and hepatitis B virus particles as detected by immunogold electron microscopy. Med Mol Morph 39: 63-71, 2006.
11. Boshuizen JA, Reimerink JH, Korteland-van Male AM, van Ham VJ, Koopmans MP, Büller HA, Dekker J and Einerhand AW: Changes in small intestinal homeostasis, morphology and gene expression during rotavirus infection of infant mice. J Virol 77: 13005-13016, 2003.

12. Li JY, Lu Y, Hu S, Sun D and Yao YM: Preventive effect of glutamine on intestinal barrier dysfunction induced by severe trauma. World J Gastroenterol 8: 168-171, 2002.

13. Yeung T, Gilbert GE, Shi J, Silvius J, Kapus A and Grinstein S: Membrane phosphatidylserine regulates surface charge and protein localization. Science 319: 210-213, 2008.

14. Yang HM, Gao J, Sheng HY, Zhou Y, Chen TX, Zhu JX and He ZJ: Effect of lactadherin on IL-2, IL-4 and INF- $\gamma$ secreted by intestinal tract of newborn rats with rotavirus infection. Guo Ji Er Ke Xue Za Zhi 36: 328-330, 2009 (In Chinese).

15. Yang LY, He ZJ and Zhu JX: The effect of lactadherin in human milk on protecting diarrhea induced by rotavirus infection. Guo Ji Er Ke Xue Za Zhi 32: 174-176, 2005 (In Chinese).

16. Zhou YJ, Gao J, Yang HM, Yuan XL, Chen TX and He ZJ: The role of the lactadherin in promoting intestinal DCs development in vivo and vitro. Clin Dev Immunol 2010: 357541, 2010.

17. Dong HT: Research of lactadherin's function. Guo Ji Er Ke Xue Za Zhi 37: 515-517, 2010 (In Chinese).

18. Li WD, Jia L, Ou Y, Huang YX and Jiang SM: Surveillance of intra-abdominal pressure and intestinal barrier function in a rat model of acute necrotizing pancreatitis and its potential early therapeutic window. PLoS One 8: e78975, 2013.

19. Shimizu K, Ogura H, Goto M, Asahara T, Nomoto K Morotomi M, Yoshiya K, Matsushima A, Sumi Y, Kuwagata Y, et al: Altered gut flora and environment in patients with severe SIRS. J Trauma 60: 126-133, 2006.

20. Vella A and Farrugia G: D-lactate acidosis: Pathologic consequence of saprophytism. Mayo Clin Proc 73: 451-456, 1998.

21. Moghaddam HS, Moghaddam HN, Kermanshahi H, Houssavi AH and Raji A: The effect of vitamin A on Mucin2 gene expression, histological and performance of broiler chicken. Global Veterinaria 5: 168-174, 2010.

22. Boshuizen JA, Reimerink JH, Korteland-van Male AM, van Ham VJ, Bouma J, Gerwig GJ, Koopmans MP, Büller HA, Dekker J and Einerhand AW: Homeostasis and function of goblet cells during rotavirus infection in mice. Virology 337: 210-221, 2005.

23. Deplancke B and Gaskins HR: Microbial modulation of innate defense: goblet cells and the intestinal mucus layer. Am J Clin Nutr 73:1131S-1141S, 2001.

24. Faure M, Moënnoz D, Montigon F, Mettraux C, Mercier S and Schiffrin EJ, Obled C, Breuillé D and Boza J: Mucin production and composition is altered in dextran sulfate sodium-induced colitis in rats. Dig Dis Sci 48: 1366-1373, 2003.

25. Sharma R, Young C and Neu J: Molecular modulation of intestinal epithelial barrier: Contribution of microbiota. J Biomed Biotechnol 2010: 305879, 2010.

26. Groschwitz KR and Hogan SP: Intestinal barrier function: Molecular regulation and disease pathogenesis. J Allergy Clin Immunol 124: 3-20, 2009.

27. Anthony $\mathrm{T}$ and Blikslager: Mucosal epithelial barrier repair to maintain pig health. Livestock Science 133: 194-199, 2010.

28. Zhou L: Progress in the pathogenesis of rotavirus-associated disease. Guo Ji Er Ke Xue Za Zhi 37: 232-234, 2010 (In Chinese).

29. Colbère-Garapin F, Martin-Latil S, Blondel B, Mousson L, Pelletier I, Autret A, François A, Niborski V, Grompone G, Catonnet $\mathrm{G}$ and van de Moer A: Prevention and treatment of enteric viral infections: Possible benefits of probiotic bacteria. Microbes Infect 9: 1623-1631, 2007.

30. Ehehalt R, Braun A, Karner M, Füllekrug J and Stremmel W: Phosphatidylcholine as a constituent in the colonic mucosal barrier: Physiological and clinical relevance. Biochim Biophys Acta 1801: 983-993, 2010.

31. Irvine EJ and Marshall JK: Increased intestinal permeability precedes the onset of Crohn's disease in a subject with familial risk. Gastroenterology 119: 1740-1744, 2000.

32. Poritz LS, Harris LR III, Kelly AA and Koltun WA: Increase in the tight junction protein claudin-1 in intestinal inflammation. Dig Dis Sci 56: 2802-2809, 2011. 